\title{
Review
}

\section{Advances in Genetic Improvement for Tocotrienol Production: A Review}

\author{
Sulaiman Rufai BABURA ${ }^{1,2}$, Siti Nor Akmar ABDULLAH ${ }^{1,3, *}$ and Huzwah KHAZA' AI ${ }^{4}$ \\ ${ }^{1}$ Department of Agriculture Technology, Faculty of Agriculture, Universiti Putra Malaysia, Malaysia \\ ${ }^{2}$ Department of Plant Biology, Bayero University Kano, Nigeria \\ ${ }^{3}$ Laboratory of Plantation Science and Technology, Institute of Plantation Studies, \\ Universiti Putra Malaysia, Malaysia \\ ${ }^{4}$ Faculty of Medicine and Health Sciences, Universiti Putra Malaysia, 43400 UPM, \\ Serdang, Selangor, Malaysia \\ (Received December 21, 2016)
}

\begin{abstract}
Summary Tocotrienols are forms of vitamin E that are present in several important food crops. Compared to tocopherols, less research has been conducted on these compounds because of their low bioavailability and distribution in plant tissues. Both tocotrienols and tocopherols are known for their antioxidant and anticancer activities, which are beneficial for both humans and animals. Moreover, tocotrienols possess certain properties which are not found in tocopherols, such as neuroprotective and cholesterol-lowering activities. The contents of tocotrienols in plants vary. Tocotrienols constitute more than $70 \%$ and tocopherols less than $30 \%$ of the total vitamin E content in palm oil, which is the best source of vitamin E. Accumulation of tocotrienols also occurs in non-photosynthetic tissues, such as the seeds, fruits and latex of some monocotyledonous and dicotyledonous plant species. The use of biotechnological techniques to increase the tocotrienol content in plants, their biological functions, and benefits to human health are discussed in this review.
\end{abstract}

Key Words tocotrienols, tocopherols, neuroprotective ability, anticancer, cholesterollowering

Vitamin E refers to a group of eight lipophilic compounds, including four tocopherols designated as $\alpha$-, $\beta$-, $\gamma$ - and $\delta$-tocopherols (Fig. 1) and their four corresponding tocotrienols (1). Evans and Bishop (1922) were the first to discover vitamin $\mathrm{E}$ as a factor in the diet of rats that helped boost their reproduction. Vitamin $\mathrm{E}$ was isolated for the first time by Gladys Emerson at the University of California, Berkeley in 1935, followed by structural elucidation by Erhard Fernholz in 1938 and its first synthesis by a team of scientists headed by Paul Karrer in 1939 (2). The antioxidant activity of vitamin E prevents lipid peroxidation propagation and is beneficial for human health (3). Vitamin $\mathrm{E}$ is widely consumed as a food supplement because it might be able to prevent or lower the morbidity and mortality rates amongst humans (4).

Tocopherols contain saturated side chains, while tocotrienols contain unsaturated side chains with double bonds at carbons 3, 7 and 11; these unsaturated side chains enable tocotrienols to efficiently penetrate tissues with saturated fatty layers, such as liver and brain tissues. Naturally occurring tocotrienols possess a chiral stereo-center at carbon 2 (Fig. 1) and 2R, 3'E, 7'E configuration. Tocotrienols possess antiangiogenic properties and 70-fold higher cellular uptake potential than

\footnotetext{
*To whom correspondence should be addressed.
}

E-mail:snaa@upm.edu.com tocopherols (5). The different forms of tocotrienols and tocopherols are distinguishable by the numbers and positions of methyl-groups attached to the benzyl rings. Tocopherols are important antioxidants that are lipid soluble. Tocopherols react with radicals produced during lipid peroxidation chain reactions, thereby protecting the organism's cell membrane from oxidation (6). Tocotrienols also possess antioxidant properties and can protect plant cells against biochemical stress, especially that resulting from unsaturated fatty acid digestion in seeds (7). Tocotrienols have not been studied as extensively as tocopherols, but researchers are now focussing on these compounds. Recent studies have shown that tocotrienols can protect neurons from damage (neurodegeneration) (7) and possess cholesterol-reducing properties (8). Moreover, oral consumption of tocotrienols can protect brain cells from damage in vivo (9). Tocotrienols are believed to have a greater ability to scavenge free radical ions and reduce peroxidation of lipids in membranes than tocopherols. Tocotrienols are produced from oil palm and rice for commercial purposes (4). The numerous beneficial properties of tocotrienols that are not observed in tocopherols have led to an interest in crops which produce tocotrienols.

Tocotrienols and tocopherols can be found in a number of plants and plant products. Palm oil contains relatively high levels of tocotrienol and tocopherol $(32-146 \mathrm{mg} / 100 \mathrm{~g})$. Vitamin $\mathrm{E}$ is also produced by 


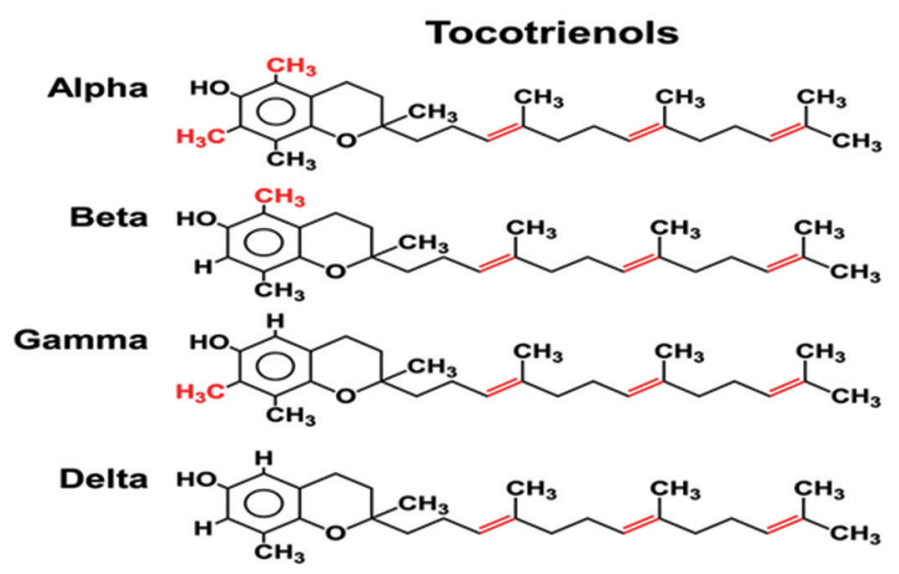

Fig. 1. Chemical structures of four forms of tocotrienols.

Table 1. Contents of tocotrienols obtained from various sources $(45,46,47)$. Each content is expressed as mg/1,000 g.

\begin{tabular}{|c|c|c|c|c|c|c|c|c|}
\hline Sources & $\alpha-\mathrm{T} 3$ & $\beta-\mathrm{T} 3$ & $\gamma-\mathrm{T} 3$ & $\delta$-T3 & $\alpha-\mathrm{T}$ & $\beta-\mathrm{T}$ & $\gamma-\mathrm{T}$ & $\delta$-T \\
\hline Palm oil & 146.0 & 32.0 & 297.0 & 80.0 & 256.0 & - & 316.0 & 70.0 \\
\hline Rice bran & 23.6 & 2.6 & 34.9 & 2.5 & 15.9 & 2.5 & 8.0 & 2.7 \\
\hline Barley & 67.0 & 12.0 & 18.6 & 2.6 & 20.1 & 1.9 & 15.1 & 4.6 \\
\hline Wheat germ & 2.6 & 8.1 & 1.8 & 0.2 & 192.0 & 65.0 & 52.3 & 0.55 \\
\hline Oat & 18.0 & - & 3.0 & - & 18.0 & 2.0 & 5.0 & 5.0 \\
\hline Coconut & 3.0 & 0.2 & 0.6 & 0.1 & 1.8 & 0.3 & 0.1 & 0.4 \\
\hline Soybean & 2.0 & 1.0 & - & - & 12.0 & 1.3 & 70.0 & 26.0 \\
\hline Palm kernel & 21 & - & - & - & 13.0 & - & - & - \\
\hline Sesame & - & - & 0.8 & 0.1 & 36.0 & 0.8 & 57.0 & 13.0 \\
\hline Adlay & 0.9 & - & 13.1 & 1.7 & & & & \\
\hline Rapeseed & 0.4 & - & 0.7 & - & 24.0 & - & 51.0 & 1.9 \\
\hline Hevea latex & 0.036 & - & 0.143 & 0.005 & & & & \\
\hline
\end{tabular}

Values are in milligrams per 1,000 g/ppm. T, tocopherols; $\mathrm{T} 3$, tocotrienols.

barley, tomato, rice bran, soybean, spinach, oat, olive, sunflower, sesame, grapeseed, coconut and wheat germ (see Table 1). It is also found in some photosynthetic microorganisms such as Synechocystis (10). Tocotrienols can be found in both edible and non-edible plant products such as in rubber latex (Table 1) (11). It has been shown that tocotrienols and tocopherols constitute $70 \%$ and $30 \%$ of total vitamin E, respectively, in palm oil. The average total content of vitamin $\mathrm{E}$ in the commercial variety of crude palm oil was found to be 600 parts per million (ppm) (12); palm oil is considered to be the best source of tocotrienols (13). The predominant isomer found in plant seeds is $\gamma$-tocopherol, while $\alpha$-tocopherol is mainly present in leaves. Tocotrienols are one of the vitamin $\mathrm{E}$ forms found in certain monocot seeds but they are not as widespread in plants as tocopherols (14). Tocotrienols and tocopherols can be present in the same plant but not necessarily in the same tissue, suggesting that their functions might differ (3).

\section{Tocotrienol Biosynthesis Pathways}

The tocotrienol and tocopherol biosynthesis pathways have a number of similarities (15); they differ only in their first committed step, where geranylgeranyl diphosphate (GGDP) is a precursor in tocotrienol biosynthesis, while phytyl diphosphate $(P D P)$ is a precur- sor for tocopherol biosynthesis $(7,10)$. Both reactions are believed to occur in the plastids (15). In the seeds of monocots, this first committed step in the biosynthesis of tocotrienols is catalysed by homogentisate geranylgeranyl transferase (HGGT) (Fig. 2). The reaction involves decarboxylation and condensation of the prenyl group of GGDP with homogentisate (HGA) to produce 2-methyl-6-geranylgeranyl-benzoquinol (3). Subsequent methylation, cyclisation and other methylation reactions that occur in the plant chloroplast of some plant species convert the product of HGGT into different tocotrienol isomers (15). HGA undergoes condensation with only PDP and not GGDP in extracts from chloroplasts of most plant species (16) to produce tocopherols. Based on research conducted on transgenic soybean and Arabidopsis thaliana, which were engineered to produce tocotrienols by the introduction of the HGGT gene, it was proposed that the enzymatic activity of HGGT is unique to the biosynthetic pathway in plants that produce tocotrienols (17).

\section{Metabolic Engineering for Tocotrienol Production and Increasing Tocotrienol Content}

The various steps involved in tocotrienol biosynthesis have been elucidated during the past two decades but the regulatory mechanism involved is still poorly 


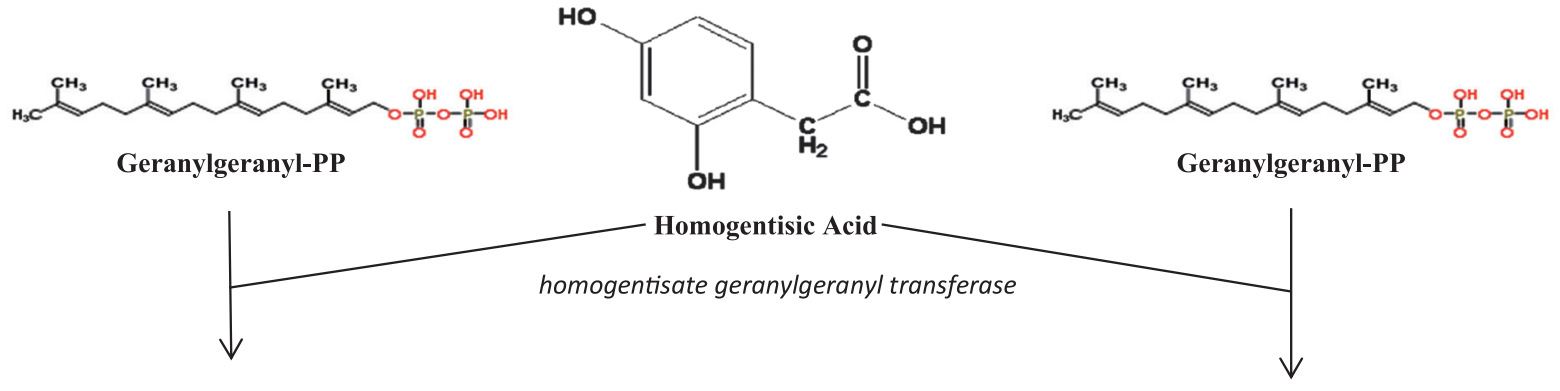

2-methyl-6- geranylgeranylbenzoquinol 2-methyl-6- geranylgeranylbenzoquinol methyltransferase $\downarrow$

\section{2,3-dimethyl-5- geranylgeranylbenzoquinol}

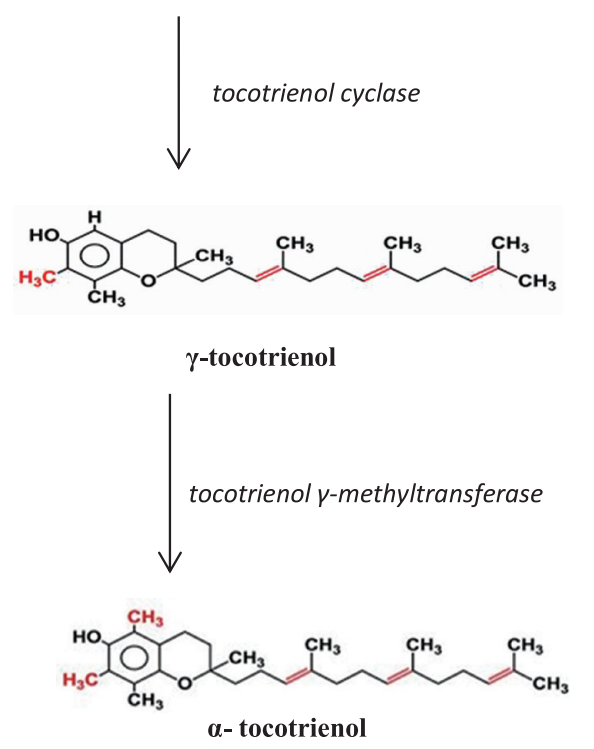

2-methyl-6- geranylgeranylbenzoquinol

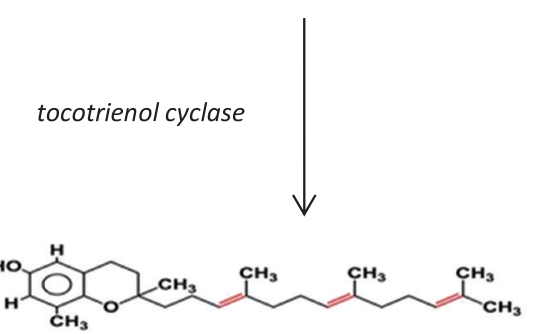

$\delta$-tocotrienol

tocotrienol $\delta$-methyltransferase

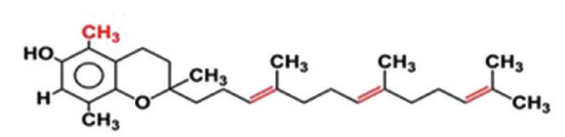

$\beta$-tocotrienol

Fig. 2. Plant tocotrienols biosynthetic pathway. The condensation of geranylgeranyldiphosphate (GGDP) and homogentisic acid catalysed by HGGT produces 2-methyl-6-geranylgeranylbenzoquinol. This serves as an intermediate that undergoes a series of methylation, cyclization and methylation reactions to form $\alpha$ - and $\gamma$-tocotrienols. $\delta$-Tocotrienol is produced when the two methylation steps are not incorporated. $\beta$-Tocotrienol is produced when only the first methylation is bypassed. Enzymes are written in italics.

understood. Research indicates that the first committed reaction catalysed by HGGT regulates and limits the metabolic flux of the isomers (18). Unlike tocopherols, tocotrienols do not frequently accumulate in plant tissues but genetic engineering can be used to enable or enhance their synthesis in transgenic plants. The expression of barley HGGT in Arabidopsis thaliana leaves led to the production of tocotrienols, which are not present in non-transgenic $A$. thaliana leaves, as well as a ten- to fifteen-fold increase in total chromanol content. Furthermore, the overexpression of HGGT from barley in corn seeds resulted in an approximately six-fold increase in tocochromanols (tocopherols and tocotrienols) $(3,4)$. The overexpression of barley HGGT in maize embryos and seeds of soybean resulted in a six- to ten-fold increase in tocochromanols, specifically tocotrienols $(10,17)$. The increase in tocochromanols was mainly because of the biosynthesis of tocotrienols. These results strongly suggest that HGGT is an important gene in the biosynthesis of tocotrienols and could be used in genetic engineering to increase antioxidant content in plants.

Research conducted to increase vitamin E content in plants includes enhancing the expression of HPT, which results in the elevation of vitamin E content, especially in soybean and canola, which are examples of typical oil crops $(16,18)$. One of the obstacles in increasing vitamin $\mathrm{E}$ in plants is the homogentisate precursor pool of the tocochromanol group. In Arabidopsis, the primary route of PDP synthesis involves desaturation of 
the geranylgeranyl group that is bound to chlorophyll rather than direct GGDP reduction by geranylgeranyl reductase (19). The phytyl moiety produced is then converted to a diphosphate following a reaction involving a kinase (19). Upregulation of Hydroxyphenyl Pyruvate Dioxygenase (HPPD) expression, which encodes an enzyme responsible for the final step of homogentisate synthesis, has also been used to enhance vitamin E content in a variety of plants. However, when it was overexpressed in transgenic plants, the results showed an insignificant increase in the overall vitamin E content $(18,19)$. Therefore, in order to increase tocotrienol or total vitamin E content, the different biosynthetic steps need to be taken into consideration.

Post-transcriptional gene silencing (PTGS) of the HPT cDNA fragment in amaranthus and leek indicated that a shift in the biochemical pathway could be used to engineer the synthesis of $\alpha$-tocotrienol. This occurs as a result of the presence of a short exogenous HPT mRNA that leads to the cleavage of both exogenous and endogenous HPT mRNA through the PTGS mechanism in plants, which reduces $\alpha$-tocopherol formation. When $\alpha$-tocopherol production is reduced to very low levels, the plant switches from the shikimate to the non-mevalonate pathway and starts producing $\alpha$-tocotrienol as a compensatory mechanism (20). Hence, the content of $\alpha$-tocochromanols (i.e., $\alpha$-tocopherol and $\alpha$-tocotrienol) in plants can be altered through PTGS by targeting their metabolic pathways.

There have been reports of unexpected accumulation of tocotrienols because of deregulated homogentisate production by an HGGT-independent pathway (without a functional $H G G T)(18,21)$. This was observed in soybean and canola seeds, which were engineered to express the A. thaliana gene, Homogentisate Dioxygenase (At-HPPD) and cynobacterial (Erwinia herbicola) prephenate dehydrogenase (Eh-TYRA) (18), and exhibited elevated HGA. The biosynthesis of tocotrienols has also been reported in transgenic tobacco leaves harboring yeast TYRA and At-HPPD (22). Moreover, when HPPD/ TYRA-expressing plants were crossed with vte2-1 HPT null mutant lacking functional HPT, the resultant plants were unable to produce tocotrienol (10). These results suggest that the production of tocotrienol in plants requires HPT and it occurs when the molar ratio of GGDP/PDP is high. This provides the genetic and molecular basis for an HGGT-independent route for tocotrienol biosynthesis.

\section{Functional Studies of Genes That Regulate Biosyn- thesis of Tocotrienols}

HGGT possesses a transit peptide that facilitates its transport to the plastid. The localization of HGGT and HPT in tissues differs; HGGT is expressed in the endosperm of seeds, while HPT is mostly expressed in the embryos of monocot seeds. Furthermore, HGGT exhibits a high degree of enzymatic activity against GGDP, but its enzymatic activity against PDP, a tocopherol side-chain precursor, is low (approximately six-fold lower). Despite the above mentioned differences, the $K_{\mathrm{m}}$ values of HGGT for the two substrates are similar (15).

The overexpression of HPT in certain plants including Arabidopsis did not result in tocotrienol production. In contrast, tocopherols can be produced in plants by the introduction of HGGT from monocots, which are known to catalyse the formation of tocotrienols. Functional studies of HGGT revealed that metabolic engineering to express HGGT in other plants enables the biosynthesis of tocotrienols in plants and organs that normally do not synthesise tocotrienols (4). Moreover, when HGGT was expressed in Arabidopsis with null mutant HPT, both tocopherols and tocotrienols were produced simultaneously. However, only tocotrienol was produced in the PDP-deficient geranylgeranyl reductase mutant in Arabidopsis. This indicates that the availability and amounts of the substrates GGDP and PDP determine the functional outcome of HGGT activity in plants (15).

\section{Regulation of Tocotrienol Biosynthesis by Promoters}

Promoters are the regulatory sequences found at the $5^{\prime}$ region of all eukaryotic genes. They regulate the level and tissue-specificity of gene expression (23). Different promoter sequences and their regulatory motifs can be identified from plant genomic sequences (24). Genes encoding enzymes in the tocopherol biosynthesis pathway have been shown to be transcriptionally activated during certain plant developmental stages in Arabidopsis. Moreover, genes in the biosynthetic pathway were upregulated by light in Arabidopsis seedlings and several cis-acting elements responsive to light were found in the promoters of these genes (21). Successful amplification of approximately $1 \mathrm{~kb}$ of promoter regions of HPT and HGGT from E. guineensis (African oil palm) using the genome-walking polymerase chain reaction (PCR) technique revealed a number of common cis-regulatory elements involved in regulating the expression of both genes. The light-responsive cis-acting elements (GATA-box, Circadian LHC and AT1-box) and elements responsive to the different phytohormones, including gibberellins, cytokinin, auxin and abscisic acid, were also identified in both promoters (25). Data also indicate that MYB transcription factors regulate proteins which are involved in tocopherol biosynthesis. Promoter motifs that serve as their binding sites are located in conserved positions within the first 200 base pairs in the promoters of the genes encoding these biosynthetic enzymes (25). Taken together, the results of the different studies suggest specific transcription factors bind the common motifs in the different genes involved in vitamin $\mathrm{E}$ biosynthesis.

Furthermore, certain transcription factors are known to influence the shikimate pathway, but the mechanism for this remains unclear. In tomato, the overexpression of the MYB transcription factor ODORANT1 led to a significant increase in phenylpropanoid content concurrently with transcriptional upregulation of several shikimate pathway genes. Furthermore, downregulation of De-etiolated 1 (DET1), which is involved in the suppression of light responses in the absence of light in tomato fruit, resulted in elevated contents of carot- 
Table 2. Biological activities of tocotrienols relative to human cellular health (48).

\begin{tabular}{|c|c|c|c|c|}
\hline & Alpha $(\alpha-)$ & $\operatorname{Beta}(\beta-)$ & $\operatorname{Gamma}(\gamma-)$ & $\operatorname{Delta}(\delta-)$ \\
\hline Heart & & & & + \\
\hline Vascular system & & & & + \\
\hline Brain & + & & & \\
\hline Free radical attack & + & + & + & + \\
\hline Cancer & & & & + \\
\hline Cholesterol reduction & & & + & + \\
\hline
\end{tabular}

enoids, flavonoids and tocopherols (26). However, systematic studies on the spatio-temporal regulatory network of gene expression and vitamin $\mathrm{E}$ contents have not been carried out. Moreover, a comparative analysis of the cis-regulatory motifs in the promoter regions of the genes of the shikimate, and tocopherol and tocotrienol pathways has not been conducted.

\section{Biological Functions of Tocotrienols}

A number of in vitro studies indicate that tocotrienols are better antioxidants than tocopherols. Tocotrienols also demonstrate high antioxidant activity in transgenic tobacco leaves (22). In the monocot endosperm, HGGT aids the synthesis of antioxidants that can extend the viability of the seed. HGGT was shown to increase the viability of artificially aged vte2-1 seeds, thereby increasing the longevity of the monocot seeds. Moreover, in Arabidopsis, the absence of tocopherols in hpt vte2-1 resulted in a decrease in seed viability because of prolonged storage and polyunsaturated fatty acid oxidation (15).

The biochemical structures of tocotrienols are similar to those of tocopherols, with the exception of the hydrocarbon tails, which bear trans-double bonds emanating from the C20 isoprenoid of the GGDP intermediate. Both tocotrienols and tocopherols possess different biological and chemical properties (27-30). Tocotrienols have neuroprotective, anticancer and cholesterollowering activities $(7,31-33)$. The medicinal properties of tocotrienols are considered to be superior than those of tocopherols (27). Among all the chemical forms of vitamin E, $\alpha$-tocopherol is regarded to have the highest antioxidant activity. However, some results indicate that tocotrienols have one of the best antioxidant activities (33). Moreover, $\alpha$-tocotrienol possesses anti-ageing properties and protects against oxidative stress (20).

The different forms of tocotrienols possess different properties and perform different functions in the human body (see Table 2); $\alpha$-tocotrienol can prevent inducible neurodegeneration (8) and was effective in treating cerebral ischemia, while $\gamma$ - and $\delta$-tocotrienol have anticancer properties (34). Oral supplements of tocotrienol protect against stroke and protect neurons against any neurotoxicity (33). These properties can be attributed to the unsaturated side chain of tocotrienols, which can penetrate into tissues that have a high content of saturated fat, such as the liver and brain, to prevent accumulation of free radicals (7).
The antitumor activities of tocotrienols and tocopherols might not be connected to their potent antioxidant properties and could result from the apoptosis and mitogenesis intracellular pathways of vitamin E (35). Oral administration of these forms of vitamins affects the immune system and production of spleen cells as well as lymphocytes of the mesenteric lymph node. Evidence suggests that some natural killer cells increased when tocotrienols were supplemented in mice. These contested MCF-7 breast cancer cells in humans (2). Research has also shown that oral administration of vitamin $\mathrm{E}$ supplements (tocotrienols and tocopherols) affects the human immune system, proliferation of spleen cells and lymphocytes (35). Plasma glucose levels as well as lipids and their peroxidation were reduced upon oral administration of tocotrienols. The mechanism by which tocotrienols promote human immunity to prevent the growth of tumour cells has been reported recently (36). However, there are safety concerns regarding the direct ingestion of vitamin E. The SELECT study reported that dietary supplements with vitamin E ( $\alpha$-tocopherol) significantly increased the risk of prostate cancer (37). Moreover, meta-analysis demonstrated that the consumption of high doses of vitamin $\mathrm{E}$ ( $\alpha$-tocopherol) could have detrimental effects and lead to increased risk of mortality (38). The use of tocotrienols from natural sources is gaining popularity (20) and there is a need to enhance tocotrienol content in plants.

Furthermore, tocotrienols can inhibit the growth and proliferation of cancerous cells and prevent angiogenesis, but the mechanism of action is yet to be discovered (37).

Shibata et al. (39) have shown that $\delta$-tocotrienol acts as an anticancer agent against human colorectal adenocarcinoma cells (DLD-1) under hypoxic and normoxic conditions. A specific dose of $\delta$-tocotrienol inhibited the growth of DLD-1 cells by inducing cell cycle arrest and apoptosis.

Among the various compounds found in whole grains, tocotrienol has the highest anticancer activity (34). This property is now the focus of preclinical and clinical trials. Moreover, $\delta$-tocotrienol was shown to have anticancer activity against pancreatic cancer (40). Besides increasing the gemcitabine activity in xenograft pancreatic cancer, $\delta$-tocotrienol also prevents the development of genetically engineered pancreatic cancer cells, prolonging the cells' lifespan (35). Treatment of pancreatic cancer cells with $\delta$-tocotrienol inhibits the growth 
of tumour cells leading to apoptosis (41). $\delta$-Tocotrienol interacts with apoptotic pathways, triggering the death of the cancer cells (42). In vitro studies on tocotrienols show that they can directly inhibit the growth of breast cancer cell lines in humans (41) and lead to apoptosis (35). This inhibitory action occurs in all cells regardless of their status (43). Among the various tocotrienols, $\gamma$ and $\delta$-tocotrienols are known to have inhibitory effects on cancer cells, which might be catalysed by the intrinsic and extrinsic metabolic pathways of tocotrienols that mediate the activation of the process. The activation of end products of extrinsic pathways by tocotrienols induces death receptors (44), which activate caspase-3 and caspase-8 (41). Additionally, tocotrienols activate intrinsic pathways that depolarise mitochondrial activities by upregulating Bax, release of cytochrome $\mathrm{C}$, cleavage of Bid and caspase-9 activation, which leads to activation of caspase-3 (40).

\section{General Outlook}

The antioxidant activities of the two forms of vitamin E, tocopherols and tocotrienols are well-known. Research is being conducted to investigate whether tocotrienols are better antioxidants than tocopherols. Furthermore, a number of investigations have been carried out to ascertain the neuroprotective, anticancer and cholesterol-lowering activities of tocotrienols.

The contents of tocotrienols in plants can be enhanced by biotechnological techniques $(4,12,31$, 32) and can be employed for the mass production of plant products that are stable against oxidation and have a long shelf life. The process can be used to produce tocotrienols with diverse therapeutic properties in oil crops (4). Transgenic expression of HGGT in A. thaliana has shown that tocotrienols can be produced in a number of plant tissues. This was the first evidence of production of tocotrienols in bioengineered plants using pathways involving HGGT. Similar achievements have been reported in other plant species, such as barley and rapeseed.

Tocotrienol biosynthesis begins with decarboxylation and condensation of the prenyl group of GGDP with HGA to produce 2-methyl-6-geranylgeranyl-benzoquinol (Fig. 2) (4, 28). The product subsequently undergoes a series of biochemical reactions to produce different forms of tocotrienols (15). For decades, research on vitamin $\mathrm{E}$ has focussed on tocopherols, while tocotrienols have been neglected. New research needs to focus on the other four forms of vitamin $\mathrm{E}(\alpha-, \beta-, \gamma$ - and $\delta$-tocotrienols) because recent findings indicate that tocotrienols could possess beneficial properties not found in tocopherols. Tocotrienols extracted from palm oil and rice are being sold as dietary supplements. Because of the critical role of HGGT in tocotrienol biosynthesis, interest in this gene is on the increase. Information regarding the promoter sequences of genes involved in vitamin E biosynthesis genes is available but the molecular mechanisms regulating tocopherol and tocotrienol biosynthesis are yet to be understood. Functional studies of the regulatory motifs in promoters of genes involved in vitamin E synthesis need to be conducted. Research on the antioxidant and free radical scavenging activities of tocochromanols shows that tocotrienols exhibit high antioxidant activity. This is because of their ability to penetrate the lipid layers of the cell membrane (7). In conclusion, tocotrienols are natural sources of antioxidants and might possess properties that are beneficial to human health.

\section{REFERENCES}

1) Valentin HE, Qi Q. 2005. Biotechnological production and application of vitamin E: Current state and prospects. Appl Microbiol Biotechnol 68: 436-444.

2) Nesaretnam K, Meganathan P, Veerasenan SD, Selvaduray KR. 2012. Tocotrienols and breast cancer: The evidence to date. Genes Nutr 7: 3-9.

3) Zhang C, Cahoon RE, Hunter SC, Chen M, Han J, Cahoon EB. 2013. Genetic and biochemical basis for alternative routes of tocotrienol biosynthesis for enhanced vitamin E antioxidant production. Plant J 73: 628-639.

4) Cahoon EB, Hall SE, Ripp KG, Ganzke TS, Hitz WD, Coughlan SJ. 2003. Metabolic redesign of vitamin E biosynthesis in plants for tocotrienol production and increased antioxidant content. Nat Biotechnol 21: 1082-1087.

5) Khosla P, Patel V, Whinter JM, Khanna S, Rakhkovskaya M, Roy S, Sen CK. 2006. Postprandial levels of the natural vitamin E tocotrienol in human circulation. Antioxid Redox Signal 8(5-6): 1059-1068.

6) Wong RSY, Radhakrishnan AK. 2012. Tocotrienol research: past into present. Nutr Rev 70: 483-490.

7) Aggarwal BB, Sundaram C, Prasad S, Kannappan R. 2010. Tocotrienols, the vitamin $\mathrm{E}$ of the 21st century: Its potential against cancer and other chronic diseases. Biochem Pharmacol 80: 1613-1631.

8) Das S, Powell SR, Wang P, Divald A, Nesaretnam K, Tosaki A, Das DK. 2005. Cardioprotection with palm tocotrienol: antioxidant activity of tocotrienol is linked with its ability to stabilize proteasomes. Am J Physiol 289: 361-367.

9) Khanna S, Patel V, Rink C, Roy S, Sen C. 2005. Delivery of orally supplemented $\alpha$-tocotrienol to vital organs of rats and tocopherol-transport protein deficient mice. Radical Biol Med 39: 310-319.

10) Zhang G-Y, Liu R-R, Xu G, Zhang P, Li Y, Tang K-X, Liu Q-Q. 2013. Increased $\alpha$-tocotrienol content in seeds of transgenic rice overexpressing Arabidopsis $\gamma$-tocopherol methyltransferase. Transgenic Res 22: 89-99.

11) Horvath G, Wessjohann L, Bigirimana J, Jansen M, Guisez Y, Caubergs R, Horemans N. 2006. Differential distribution of tocopherols and tocotrienols in photosynthetic and non-photosynthetic tissues. Phytochemistry 67: 1185-1195.

12) $\mathrm{Ng} \mathrm{MH}$, Choo YM, Ma AN, Chuah $\mathrm{CH}$, Hashim MA. 2004. Separation of vitamin E (tocopherol, tocotrienol, and tocomonoenol) in palm oil. Lipids 39: 1031-1035.

13) Maarasyid C, Muhamad II, Supriyanto E. 2014. Potential source and extraction of vitamin $\mathrm{E}$ from palm-based oils: A review. Jurnal Teknologi 4: 43-50.

14) Ahsan H, Ahad A, Siddiqui WA. 2015. A review of characterization of tocotrienols from plant oils and foods. $J$ Chem Biol 8: 45-59.

15) Yang W, Cahoon RE, Hunter SC, Zhang C, Han J, Borgschulte T, Cahoon EB. 2011. Vitamin E biosynthesis: 
functional characterization of the monocot homogentisate geranylgeranyl transferase. Plant J 65: 206-217.

16) Collakova E, DellaPenna D. 2001. Isolation and functional analysis of homogentisate phytyltransferase from Synechocystis sp. PCC 6803 and Arabidopsis. Plant Physiol 127: 1113-1124.

17) Cahoon EB, Dietrich CR, Meyer K, Damude HG, Dyer JM, Kinney AJ. 2006. Conjugated fatty acids accumulate to high levels in phospholipids of metabolically engineered soybean and Arabidopsis seeds. Phytochemistry 67: 1166-1176.

18) Karunanandaa B, Qi Q, Hao M, Baszis SR, Jensen PK, Wong Y-HH, Valentin HE. 2005. Metabolically engineered oilseed crops with enhanced seed tocopherol. Metab Engineer J 7: 384-400.

19) Valentin HE, Lincoln K, Moshiri F, Jensen PK, Qi Q, Venkatesh TV, Last RL. 2006. The Arabidopsis vitamin E pathway gene 5-1 mutant reveals a critical role for phytol kinase in seed tocopherol biosynthesis. Plant Cell 18: 212-224.

20) Munusamy U, Abdullahi SNA, Aziz MA, Khaza'ai H. 2015. Metabolic engineering of $\alpha$-tocotrienol through PTGS mechanisms and isoprenoid/non-mevalonate pathways in perennial crops. Plant Cell Biotech Mol Biol 16(3-4): 119-129.

21) Cordoba E, Salmi M, León P. 2009. Unravelling the regulatory mechanisms that modulate the MEP pathway in higher plants. J Exp Bot 60: 2933-2943.

22) Abbasi A-R, Saur A, Hennig P, Tschiersch H, Hajirezaei M, Hofius D, Voll LM. 2009. Tocopherol deficiency in transgenic tobacco (Nicotiana tabacum L.) plants leads to accelerated senescence. Plant Cell Environ 32: 144-157.

23) Hernandez-Garcia CM, Finer JJ. 2014. Identification and validation of promoters and cis-acting regulatory elements. Plant Sci 218: 109-119.

24) Rushton PJ. 2002. Synthetic plant promoters containing defined regulatory elements provide novel insights into pathogen- and wound-induced signaling. Plant Cell J 14: 749-762.

25) Kong SL, Abdullah SNA, Ho CL, Amiruddin MD. 2016. Molecular cloning, gene expression profiling and in silico sequence analysis of vitamin E biosynthetic genes from the oil palm. Plant Gene 5: 100-108.

26) Enfissi EMA, Barneche F, Ahmed I, Lichtlé C, Gerrish C, McQuinn RP, Fraser PD. 2010. Integrative transcript and metabolite analysis of nutritionally enhanced DEETIOLATED1 downregulated tomato fruit. Plant Cell 22: 190-215.

27) Sen CK, Khanna S, Roy S. 2006. Tocotrienols: Vitamin E beyond tocopherols. Life Sci 78: 2088-2098.

28) Hunter SC, Cahoon EB. 2007. Enhancing vitamin E in oilseeds: unraveling tocopherol and tocotrienol biosynthesis. Lipids 42: 97-108.

29) Falk J, Munné-Bosch S. 2010. Tocochromanol functions in plants: antioxidation and beyond. J Exp Bot 61: 1549-1566.

30) Prasad K. 2011. Tocotrienols and cardiovascular health. Current Pharm Design 17: 2147-2154.

31) Qureshi AA, Sami SA, Salser WA, Khan FA. 2002. Dosedependent suppression of serum cholesterol by tocotrienol-rich fraction (TRF25) of rice bran in hypercholesterolemic humans. Atherosclerosis 161: 199-207.

32) Theriault A, Chao J-T, Wang Q, Gapor A, Adeli K. 1999. Tocotrienol: a review of its therapeutic potential. Clin
Biochem 32: 309-319.

33) Khanna S, Roy S, Parinandi NL, Maurer M, Sen CK. 2006. Characterization of the potent neuroprotective properties of the natural vitamin E alpha-tocotrienol. J Neurochem 98: 1474-1486.

34) Kannappan R, Gupta SC, Kim JH, Aggarwal BB. 2012. Tocotrienols fight cancer by targeting multiple cell signaling pathways. Genes Nutr 7: 43-52.

35) Husain K, Centeno BA, Chen D-T, Hingorani SR, Sebti SM, Malafa MP. 2013. Vitamin E $\delta$-tocotrienol prolongs survival in the LSL-KrasG12D/ + ;LSL-Trp53R172H/ +;Pdx1-Cre (KPC) transgenic mouse model of pancreatic cancer. Cancer Prev Res 6: 1074-1083.

36) Ahsan H, Ahad A, Iqbal J, Siddiqui WA. 2014. Pharmacological potential of tocotrienols: a review. Nutr Metabol 11: 52.

37) Klein EA, Thompson IM, Tangen CM, Crowley JJ, Lucia MS, Goodman PJSG. 2011. Vitamin E and the risk of prostate cancer. JAMA 306: 1549-1556.

38) Miller ER, Pastor-Barriuso R, Dalal D, Riemersma RA, Appel LJ, Guallar E. 2005. Meta-analysis: High-dosage vitamin E supplementation may increase all-cause mortality. Ann Intern Med 142: 37-46.

39) Shibata A, Nakagawa K, Tsuduki T, Miyazawa T. 2015. $\delta$-Tocotrienol treatment is more effective against hypoxic tumor cells than normoxic cells: potential implications for cancer therapy. J Nutr Biochem 26: 832-840.

40) Wang C, Husain K, Zhang A, Centeno BA, Chen D, Tong Z, Malafa MP. 2015. ScienceDirect EGR-1/Bax pathway plays a role in vitamin $\mathrm{E} \delta$-tocotrienol-induced apoptosis in pancreatic cancer cells. J Nutr Biochem 26: 797-807.

41) Hodul PJ, Dong Y, Husain K, Pimiento JM, Chen J, Zhang A, Malafa MP. 2013. Vitamin E $\delta$-tocotrienol induces p27(Kip1)-dependent cell-cycle arrest in pancreatic cancer cells via an E2F-1-dependent mechanism. PloS One 8: $521-526$.

42) Wang W, Liu J. 2015. Genome-wide identification and expression analysis of the polyamine oxidase gene family in sweet orange (Citrus sinensis). Gene 555: 421-429.

43) Bai J, Sui J, Demirjian A, Vollmer CM, Marasco W, Callery MP. 2005. Predominant Bcl-XL knockdown disables antiapoptotic mechanisms: tumor necrosis factor-related apoptosis-inducing ligand-based triple chemotherapy overcomes chemoresistance in pancreatic cancer cells in vitro. Cancer Res 65: 2344-2352.

44) Park SK, Sanders BG, Kline K. 2010. Tocotrienols induce apoptosis in breast cancer cell lines via an endoplasmic reticulum stress-dependent increase in extrinsic death receptor signaling. Breast Cancer Res Treat 124: 361-375.

45) Hassanien MMM, Abdel-Razek AG, Rudzińska M, Siger A, Ratusz K, Przybylski R,. 2014. Phytochemical contents and oxidative stability of oils from non-traditional sources. Eur J Lipid Sci Technol 116: 1563-1571.

46) Sajari R, Abd Razak NH, Yusof F, Arif SAM, Perkins M, Yeang HY. 2014. Improved efficiency of tocotrienol extraction from fresh and processed latex. J Rubber Res 17: 245-260.

47) Shahidi F, de Camargo A. 2016. Tocopherols and tocotrienols in common and emerging dietary sources: Occurrence, applications, and health benefits. Int J Mol Sci 17: 1745.

48) Toldrá F, Paliyath G, Hui YH, Simpson BK, Nollet LML, Benjakul S. 2012. Food Biochemistry and Food Processing, p 1724. Wiley, Chichester. 